Research Article

\title{
Decomposition of Topologies Which Characterize the Upper and Lower Semicontinuous Limits of Functions
}

\author{
Agata Caserta \\ Department of Mathematics, SUN, 81100 Caserta, Italy \\ Correspondence should be addressed to Agata Caserta, agata.caserta@unina2.it
}

Received 20 June 2011; Accepted 23 August 2011

Academic Editor: Ljubisa Kocinac

Copyright (C) 2011 Agata Caserta. This is an open access article distributed under the Creative Commons Attribution License, which permits unrestricted use, distribution, and reproduction in any medium, provided the original work is properly cited.

We present a decomposition of two topologies which characterize the upper and lower semicontinuity of the limit function to visualize their hidden and opposite roles with respect to the upper and lower semicontinuity and consequently the continuity of the limit. We show that (from the statistical point of view) there is an asymmetric role of the upper and lower decomposition of the pointwise convergence with respect to the upper and lower decomposition of the sticking convergence and the semicontinuity of the limit. This role is completely hidden if we use the whole pointwise convergence. Moreover, thanks to this mirror effect played by these decompositions, the statistical pointwise convergence of a sequence of continuous functions to a continuous function in one of the two symmetric topologies, which are the decomposition of the sticking topology, automatically ensures the convergence in the whole sticking topology.

\section{Introduction}

Since the end of the nineteenth century several outstanding papers appeared to formulate a set of conditions, which are both necessary and sufficient, to be added to pointwise convergence of a sequence of continuous functions, to preserve continuity of the limit. Indeed, all classical kinds of convergences of sequences of functions between metric spaces (Dini, Arzelà, Alexandroff) are based on the pointwise convergence assumption that has been always considered a preliminary one. Recently, in [1,2], Caserta et al. proposed a new model to investigate convergences in function spaces: the statistical one. Actually, they obtained results parallel to the classical ones, concerning the continuity of the limit, in spite of the fact that statistical convergence has a minor control of the whole set of functions. In [2] they proved that continuity of the limit of a sequence of functions is equivalent to several modes of statistical convergence which are similar, but weaker than the classical ones. A parallel to the classical results is expected since, after all, in [3] the authors found the statistical convergence 
to be the same as a very special regular triangular matrix summability method for bounded (and some unbounded) sequences. Thus many new results concerning statistical convergence follow from the corresponding known results for matrix summability.

In 1969 Bouleau $[4,5]$ defined the sticking topology as the weakest topology finer than pointwise convergence to preserve continuity. In [6], Beer presented two new topologies on $\mathbb{R}^{X}$, finer than the topology of pointwise convergence, which are indeed the decomposition of the sticking topology in upper and lower halves. For its upper part the closure of $C(X)$ is the set of all upper semicontinuous functions, and the pointwise convergence of a sequence in $C(X)$ to an upper semicontinuous limit automatically ensures convergence in this finer topology. Beer also pointed out that the pointwise convergence within the set of all upper semicontinuous functions does not ensure this convergence, even if the limit is continuous.

We realize that the pointwise convergence is a too strong assumption to analyze the upper and lower semicontinuous limit of functions, and as a result continuity, in fact the whole pointwise convergence conceals the properties that functions and limits must satisfy. We offer a decomposition of the pointwise convergence in upper and lower halves that will enable us to visualize their hidden and opposite roles with respect to the upper and lower semicontinuity of limits and functions. We introduce two new properties for a sequence of functions with respect to a prospective limit function and give a characterization of upper (resp., lower) semicontinuity of the limit in terms of this new properties of the whole sequence.

The decomposition of the pointwise convergence is necessary to expose the salient features of these new important properties and display the asymmetric role of the upper and lower decomposition of the topology of pointwise convergence with respect to the upper and lower decomposition of the sticking topology. This phenomenon is completely concealed if we consider the whole pointwise convergence within the set of all continuous functions.

Thanks to a careful analysis of the behavior of this decomposition and its interplay with upper and lower semicontinuity of functions and limits, we can extend the results in [2]. We prove that whole statistical pointwise convergence in $C(X)$ ensures convergence in the two symmetric topologies of the decomposition of the sticking topology, and therefore the statistical pointwise convergence of a sequence of continuous functions to a continuous function forces the convergence in a wider class of finer topologies.

\section{Preliminaries}

Let $(X, d)$ be metric space. We denote the power set of $X$ by $p(X)$, the nonempty (resp., nonempty finite) subsets of $X$ by $p_{0}(X)$ (resp., $F_{0}(X)$ ). Let $X$ be topological space, $C(X)$ denotes the set of all real valued continuous functions on $X$. Recall that a function $f \in \mathbb{R}^{X}$ is upper (resp., lower) semicontinuous at $x \in X$ if for every $\epsilon>0$ there is $\delta>0$ such that for all $y \in S(x, \delta)$ then $f(y)<f(x)+\epsilon$ (resp., $f(y)>f(x)-\epsilon)$. Also $f$ is upper semicontinuous if it is upper semicontinuous at every $x \in X$. By $\mathcal{L}(X)$ (resp., $\mathcal{U}(X))$ we denote the set of all real valued lower (resp., upper) semicontinuous functions defined on $X$; evidently $\mathfrak{u}(X) \cap \mathcal{L}(X)=$ $C(X)$.

A quas-iuniformity for a set $X$ is a collection reflexive relations $\mathcal{U}$ on $X$ that forms a filter and such that for every $U \in \mathcal{U}$ there is $V \in \mathcal{U}$ such that $V \circ V \subseteq U$. The pair $(X, \mathcal{U})$ is called quasi-uniform space. A subfamily $\hat{U}$ of $\mathcal{U}$ is a base for the quasi-uniformity $\mathcal{U}$ if it is cofinal in $\mathcal{U}$ with respect to the inclusion; that is, for every $U \in \mathcal{U}$, there is $\widehat{U} \in \hat{\mathcal{U}}$ such that $\widehat{U} \subseteq U$.

If $\mathcal{U}$ is a quasi-uniformity for $X$, then $\mathcal{U}^{\prime}=\left\{U^{-1}: U \in \mathcal{U}\right\}$ where $U^{-1}=\{(x, y)$ : $(y, x) \in U\}$, is also a quasi-uniformity for $X$. The collections $\mathcal{U}$ and $\mathcal{U}^{\prime}$ are called conjugate quasi-uniformities [7]. 
The quasi-uniform space $(X, \mathcal{U})$ will always be considered to be a topological space with the topology obtained by using as the family of all neighborhoods of a point $x_{0} \in X$ all sets of the form $U\left(x_{0}\right)=\left\{x \in X:\left(x_{0}, x\right) \in U\right\}$, where $U$ runs over $\mathcal{U}$. Such a topology is called topology of the quasi-uniformity. Note that each topology on $X$ is induced by a quasi-uniformity (see $[8,9]$ ), the most familiar of which is the Pervin quasi-uniformity [8].

A quasi-uniform space $(X, \mathfrak{U})$ is said to be a uniform space, and the family $\mathfrak{U}$ will be called a uniformity for $X$, if and only if the quasi-uniformity satisfies the symmetric relation: if $U \in U$, then $U^{-1} \in \mathcal{U}$. For a quasi-uniformity $\mathcal{U}$ the smallest uniformity containing $\mathcal{U}$ has a base all sets of the form $\widehat{U} \cap \widehat{U^{-1}}$ where $\widehat{U}$ runs over a prescribed base for $\mathcal{U}$. Moreover, a topology is induced by a uniformity if and only if the space is completely regular $[7,10]$.

Let us familiarize the reader with the notion of statistical convergence, that first appeared in 1935 under the name of almost convergence in the celebrated monograph of Zygmund [11]. The definition of statistical convergence for sequences of real numbers was given by Fast in [12] and is based on the notion of asymptotic density of a subset of natural numbers. Let $A \subset \mathbb{N}$ and $n \in \mathbb{N}$. Put $A(n):=\{k \in A: k \leq n\}$. Then one defines

$$
\begin{aligned}
& \underline{\partial}(A):=\liminf _{n \rightarrow \infty} \frac{|A(n)|}{n}, \\
& \bar{\partial}(A):=\limsup _{n \rightarrow \infty} \frac{|A(n)|}{n},
\end{aligned}
$$

as the lower and upper asymptotic density of $A$, respectively. If $\underline{\partial}(A)=\bar{\partial}(A)$, then

$$
\partial(A)=\lim _{n \rightarrow \infty} \frac{|A(n)|}{n}
$$

is the asymptotic (or natural) density of $A$.

All the three densities, if they exist, are in $[0,1]$. We recall also that $\partial(\mathbb{N} \backslash A)=1-\partial(A)$ for $A \subset \mathbb{N}$. A set $A \subset X$ is said to be statistically dense if $\partial(A)=1$. Let us mention that the union and intersection of two statistically dense sets in $\mathbb{N}$ are also statistically dense. For additional properties of the asymptotic density, in a more general setting, the reader might consult [13].

A sequence $\left(x_{n}\right)_{n \in \mathbb{N}}$ in a topological space $X$ is said to converge statistically (or shortly, st-converge ) to $x \in X$, if for every neighborhood $U$ of $x, \partial\left(\left\{n \in \mathbb{N}: x_{n} \notin U\right\}\right)=0$. This will be denoted by $\left(x_{n}\right)_{n \in \mathbb{N}} \stackrel{\text { st- } \tau}{\rightarrow} x$, where $\tau$ is a topology on $X$.

It was shown $[14]($ see $[15,16]$ for $X=\mathbb{R})$ that for first countable spaces this definition is equivalent to the statement: there exists a subset $A$ of $\mathbb{N}$ with $\partial(A)=1$ such that the sequence $\left(x_{n}\right)_{n \in A}$ converges to $x$. Recently in [17], Çakalli and Khan pointed out that the first countability is not a necessary condition.

\section{Decomposition of Pointwise Convergence and Weakly Exhaustiveness in $\mathbb{R}^{X}$}

Given $F \in \mathcal{F}_{0}(X)$ and $\epsilon>0$, a base for the standard uniformity for the topology of pointwise convergence $\tau_{p}$ on $\mathbb{R}^{X}$ consists of all entourages of the form

$$
[F ; \epsilon]_{p}:=\{(f, g): \forall x \in F|f(x)-g(x)|<\epsilon\}
$$


In what follows, we offer a decomposition of the pointwise convergence in upper and lower part to better visualize their hidden and opposite roles with respect to the lower and upper semicontinuity of limits and functions.

Definition 3.1. Let $(X, d)$ be metric space, $F \in F_{0}(X)$, and $\epsilon>0$. Consider the quasi-uniformity on $\mathbb{R}^{X}$ having as a base all sets of the form

$$
[F ; \epsilon]_{p}^{u}:=\{(f, g): \forall x \in F f(x)<g(x)+\epsilon\}
$$

The induced upper pointwise topology on $\mathbb{R}^{X}$ is denoted by $\tau_{p}^{u}$.

The conjugate quasi-uniformity has as a base all sets

$$
[F ; \epsilon]_{p}^{l}:=\{(f, g): \forall x \in F f(x)>g(x)-\epsilon\}
$$

We denote the induced lower pointwise topology on $\mathbb{R}^{X}$ by $\tau_{p}^{l}$. Clearly the topology of pointwise convergence $\tau_{p}$ can be written as $\tau_{p}=\tau_{p}^{u} \vee \tau_{p}^{l}$.

In the next definition we also give a decomposition of the statistical weakly exhaustive property for a sequence of functions. Statistical weakly exhaustiveness is a variation of the classical definition of weakly exhaustiveness [18] and has been deeply investigated in $[1,2]$.

Definition 3.2. A sequence $\left(f_{n}\right)_{n \in \omega}$ in $\mathbb{R}^{X}$ is said to be cofinally upper (resp., lower) weakly exhaustive at $x_{0} \in X$, simply cf-upper (resp., lower) weakly exhaustive at $x_{0}$, if for every $\epsilon>0$ there is $\delta>0$ such that for all $y \in S\left(x_{0}, \delta\right)$ there exists a cofinal subset $\left\{n_{y_{k}}\right\}_{k}$ of $\mathbb{N}$ such that for all $n \in\left\{n_{y_{k}}\right\}_{k}$ we have $f_{n}(y)<f_{n}\left(x_{0}\right)+\epsilon$ (resp., $\left.f_{n}(y)>f_{n}\left(x_{0}\right)-\epsilon\right)$. The sequence $\left(f_{n}\right)_{n \in \omega}$ is cf-upper weakly exhaustive if it is cf-upper weakly exhaustive at every $x_{0} \in X$.

In case $\left\{n_{y_{k}}\right\}_{k}$ is statistically dense in $\mathbb{N}$ we say that $\left(f_{n}\right)_{n \in \omega}$ is statistically upper (resp., lower) weakly exhaustive at $x_{0} \in X$, shortly st-upper (resp., lower) weakly exhaustive at $x_{0}$.

We point out that upper (resp., lower) weakly exhaustiveness are weaker notion than what is called equisemicontinuity, in that the cofinal set of indexes depends on the point $y$.

We now introduce two new properties for a sequence of real valued functions $\left(f_{n}\right)_{n \in \omega}$ with respect to a prospective limit function $f$ at a point that play a significant role in our investigation (see Theorem 4.9).

Definition 3.3. Let $\left(f_{n}\right)_{n \in \omega}, f$ in $Y^{X}$, and $x_{0} \in X$. The sequence $\left(f_{n}\right)_{n \in \omega}$ is said to be cofinally almost below (resp., above) $f$ around $x_{0} \in X$, shortly cf-almost below (resp., above) $f$ around $x_{0}$; if for every $\epsilon>0$ there is $\delta>0$ such that for all $y \in S\left(x_{0}, \delta\right)$ there exists a cofinal subset $\left\{n_{y_{k}}\right\}_{k}$ of $\mathbb{N}$ such that for all $n \in\left\{n_{y_{k}}\right\}_{k}$ we have $f_{n}(y)<f\left(x_{0}\right)+\epsilon$ (resp., $\left.f_{n}(y)>f\left(x_{0}\right)-\epsilon\right)$.

When $\left\{n_{y_{k}}\right\}_{k}$ is statistically dense in $\mathbb{N}$ we say that $\left(f_{n}\right)_{n \in \omega}$ is statistically almost below (resp., above) $f$ around $x_{0} \in X$, shortly st-almost below (resp., above) $f$ around $x_{0}$.

In the next propositions we show that each of the notions cf-weakly exhaustive at $x_{0}$ and cf-almost below $f$ around $x_{0}$ are forced by one of the $\tau_{p}^{u}$-convergence and $\tau_{l}^{u}$-convergence and the other property.

Proposition 3.4. Let $\left(f_{n}\right)_{n \in \omega}$ be a sequence of functions in $\mathbb{R}^{X}$ that is $\tau_{p}^{u}$-convergent to $f \in \mathbb{R}^{X}$. If the sequence $\left(f_{n}\right)_{n \in \omega}$ is cf-almost below $f$ around $x_{0}$, then 
(i) $\left(f_{n}\right)_{n \in \omega}$ is cf-upper weakly exhaustive at $x_{0}$, and

(ii) $f$ is upper semicontinuous at $x_{0}$.

Proof. (i) Let $\epsilon>0$ be fixed. Since $\left(f_{n}\right)_{n \in \omega}$ is $\tau_{p}^{u}$-convergent to $f$ at $x_{0}$, there exists $n_{1}$ such that for all $n \geq n_{1}$ we have that $f_{n} \in\left[\left\{x_{0}\right\}, \epsilon / 4\right]_{p}^{u}(f)$; hence for every $n \geq n_{1}, f\left(x_{0}\right) \leq f_{n}\left(x_{0}\right)+\epsilon / 4$. Since $\left(f_{n}\right)_{n \in \omega}$ is cf-almost below $f$ around $x_{0}$, there is a $\delta_{0}>0$ such that for every $y \in S\left(x_{0}, \delta_{0}\right)$ there exists $\left\{n_{y_{k}}\right\}_{k}$ cofinal in $\mathbb{N}$, with $n_{y_{k}} \geq n_{1}$ for every $k \in \omega$, and for all $n \in\left\{n_{y_{k}}\right\}, f_{n}(y) \leq$ $f\left(x_{0}\right)+\epsilon / 4$. Thus for all $n \in\left\{n_{y_{k}}\right\}$ and $y \in S\left(x_{0}, \delta_{0}\right)$ we have $f_{n}(y) \leq f\left(x_{0}\right)+\epsilon / 4 \leq f_{n}\left(x_{0}\right)+$ $\epsilon / 2$.

(ii) Let $\epsilon>0$ be fixed. Since $\left(f_{n}\right)_{n \in \omega}$ is cf-almost below $f$ around $x_{0}$, there is a $\delta_{0}>0$ such that for every $y \in S\left(x_{0}, \delta_{0}\right)$ there exists $\left\{n_{y_{k}}\right\}_{k}$ cofinal in $\mathbb{N}$ and for all $n \in\left\{n_{y_{k}}\right\}, f_{n}(y) \leq$ $f\left(x_{0}\right)+\epsilon / 2$. Since $\left(f_{n}\right)_{n \in \omega}$ is $\tau_{p}^{u}$-convergent to $f$ at $y$, there exists $n_{y}$ such that for all $n \geq n_{y}$ we have that $f(y) \leq f_{n}(y)+\epsilon / 2$. By cofinality of the index set, let $n \in\left\{n_{y_{k}}\right\}$ be such that $n \geq n_{y}$. It follows that $f(y) \leq f_{n}(y)+\epsilon / 2<f\left(x_{0}\right)+\epsilon$.

Proposition 3.5. Let $\left(f_{n}\right)_{n \in \omega}$ be a sequence of functions in $\mathbb{R}^{X}$, that is, $\tau_{p}^{l}$-convergent to $f \in \mathbb{R}^{X}$. If $\left(f_{n}\right)_{n \in \omega}$ is cf-upper weakly exhaustive at $x_{0}$, then it is cf-almost below $f$ around $x_{0}$.

Proof. Let $\epsilon>0$ be fixed. By assumption there is a $\delta>0$ such that for every $y \in S\left(x_{0}, \delta\right)$ there is $\left\{n_{y_{k}}\right\}_{k}$ cofinal in $\mathbb{N}$ and for all $n \in\left\{n_{y_{k}}\right\}, f_{n}(y) \leq f_{n}\left(x_{0}\right)+\epsilon / 4$. Since $\left(f_{n}\right)_{n \in \omega}$ is $\tau_{p}^{l}$-convergent to $f$ at $x_{0}$, there exists $n_{0}$ such that for all $n \geq n_{0}, f_{n}\left(x_{0}\right) \leq f\left(x_{0}\right)+\epsilon / 4$. It follows that for all $n \in\left\{n_{y_{k}}\right\}_{k}$ with $n \geq n_{0}, f_{n}(y) \leq f_{n}\left(x_{0}\right)+\epsilon / 4 \leq f\left(x_{0}\right)+\epsilon / 2$.

Proposition 3.6. Let $\left(f_{n}\right)_{n \in \omega}$ be a sequence of functions in $\mathbb{R}^{X}$. If $\left(f_{n}\right)_{n \in \omega}$ is $\tau_{p}^{l}$-convergent to $f \in \mathbb{R}^{X}$ and $f$ is upper semicontinuous at $x_{0}$, then $\left(f_{n}\right)_{n \in \omega}$ is cf-almost below $f$ around $x_{0}$.

Proof. Let $\epsilon>0$ be fixed, there is a $\delta_{0}>0$ such that for every $y \in S\left(x_{0}, \delta_{0}\right), f(y)<f\left(x_{0}\right)+\epsilon / 2$. Since $\left(f_{n}\right)_{n \in \omega}$ is $\tau_{p}^{l}$-convergent to $f$ at $y$, there exists $n_{y}$ such that for all $n \geq n_{y}, f_{n}(y) \leq$ $f(y)+\epsilon / 2$. Thus for every $y \in S\left(x_{0}, \delta_{0}\right)$, the subset $\left\{n_{y_{k}}\right\}_{k}=\left\{n: n \geq n_{y}\right\}$ is cofinal in $\mathbb{N}$ and $f_{n}(y)<f(y)+\epsilon / 2<f\left(x_{0}\right)+\epsilon$.

Therefore, if we assume the whole pointwise convergence the two properties, cf-upper weakly exhaustive at $x_{0}$ and cf-almost below $f$ around $x_{0}$, associated to the upper and lower pointwise convergence, coincide.

Corollary 3.7. Let $\left(f_{n}\right)_{n \in \omega}$ be a sequence of functions in $\mathbb{R}^{X}$ that is pointwise convergent to $f \in \mathbb{R}^{X}$ and $x_{0} \in X$. The following are equivalent:

(i) $f$ is upper semicontinuous at $x_{0}$,

(ii) $\left(f_{n}\right)_{n \in \omega}$ is cf-upper weakly exhaustive at $x_{0}$,

(iii) $\left(f_{n}\right)_{n \in \omega}$ is cf-almost below $f$ around $x_{0}$.

All the previous propositions hold if we consider statistical convergences of the sequences of functions. Therefore, next corollary, similar to that in [1], improves the result weakening the condition on the sequence.

Corollary 3.8. Let $\left(f_{n}\right)_{n \in \omega}$ be a sequence of functions in $\mathbb{R}^{X}$, that is, st- $\tau_{p}$ convergent to $f \in \mathbb{R}^{X}$. The sequence $\left(f_{n}\right)_{n \in \omega}$ is cf-weakly exhaustive at $x_{0}$ if and only if $f$ is continuous at $x_{0}$. 


\section{Decomposition of Sticking Topology in $C(X)$}

First, let us recall some definitions and results given in [6].

Definition 4.1. Let $(X, d)$ be metric space, $F \in \mathcal{F}_{0}(X)$, and $\epsilon>0$. Consider the quasi-uniformity on $\mathbb{R}^{X}$ having as a base all sets of the form

$$
\begin{aligned}
{[F ; \epsilon]^{u}:=} & \{(f, g): \forall x \in F|f(x)-g(x)|<\epsilon, \exists \text { a neighborhood } V \text { of } F \\
& \text { such that } \forall x \in V, g(x)<f(x)+\epsilon\} .
\end{aligned}
$$

We denote the induced topology on $\mathbb{R}^{X}$ as $\tau^{u}$. Also we denote by $\tau^{l}$ the topology induced on $\mathbb{R}^{X}$ by the conjugate quasi-uniformity. If we take the uniformity generated by our standard uniformity for $\tau^{u}$ and its conjugate $\tau^{l}$ we get a uniformity having as a base all sets of the form

$$
\begin{array}{r}
{[F ; \epsilon]^{\square}:=\{(f, g): \exists \text { a neighborhood } V \text { of } F \text { such that } \forall x \in V,|f(x)-g(x)|<\epsilon\}} \\
\left(F \in \mathcal{F}_{0}(X), \epsilon>0\right) .
\end{array}
$$

The topology $\tau_{\square}$ induced by this uniformity is called the sticking topology by Bouleau in $[4,5]$ and the topology of strong pointwise convergence by Beer and Levi in [19]. This topology has the intrinsic property to preserve continuity: $C(X)$ is $\tau_{\square}$-closed in $\mathbb{R}^{X}$, and the $\tau_{\square}$ convergence reduces to pointwise convergence on $C(X)$ itself. In [20] the authors gave a complete characterization of continuity for the pointwise limit of continuous functions. In $[1,2]$, it has been proved that similar results about continuity of the limit function are true for statistical pointwise convergence of sequences of functions between metric spaces.

In [1], a statistical version of the classical Alexandroff convergence, introduced in 1948 in [21] (see [20]), and the well-known quasi-uniform convergence, introduced by Arzelà [22] in 1883 and extended by Bartle [23] in 1955, were defined (see also [20]).

Definition 4.2. A sequence $\left(f_{n}\right)_{n \in \omega}$ in $C(X, Y)$ is said to be statistically Alexandroff convergent to $f \in Y^{X}$, denoted by $\left(f_{n}\right)_{n \in \omega}$ being st- $\tau_{\mathrm{Al}}$, provided $\left(f_{n}\right)_{n \in \omega} \stackrel{\text { st }-\tau_{p}}{\rightarrow} f$ and for every $\epsilon>0$ and every statistically dense set $A \subset \mathbb{N}$ there exist an infinite set $M_{A}=\left\{n_{1}<n_{2}<\cdots n_{k}<\cdots\right\} \subset A$ and an open cover $\mathcal{U}=\left\{U_{n}: n \in A\right\}$ such that for every $x \in U_{k}$ we have $\rho\left(f_{n_{k}}(x), f(x)\right)<\epsilon$.

Definition 4.3. A sequence $\left(f_{n}\right)_{n \in \omega}$ in $C(X, Y)$ is said to be statistically Arzelà convergent to $f \in$ $Y^{\mathrm{X}}$, denoted by $\left(f_{n}\right)_{n \in \omega}$ being st- $\tau_{\mathrm{Arz}}$ convergent to $f$, if $\left(f_{n}\right)_{n \in \omega}$ is st- $\tau_{p}$ convergent to $f$, and for every $\epsilon>0$ and every statistically dense set $A \subset \mathbb{N}$ there exists a finite set $\left\{n_{1}, n_{2}, \ldots, n_{k}\right\} \subset$ $A$ such that for every $x \in X$ it holds $\rho\left(f_{n_{i}}(x), f(x)\right)<\epsilon$ for at least one $i \leq k$.

In [2] (Theorem 2.3) the authors proved the following characterization for the continuity of a statistical pointwise limit of continuous functions.

Theorem 4.4 (see [2]). Let $\left(f_{n}\right)_{n \in \omega}$ be a sequence of functions in $C(X)$, that is, st- $\tau_{p}$ convergent to $f \in \mathbb{R}^{X}$. The following are equivalent:

(i) $f$ is continuous,

(ii) $\left(f_{n}\right)_{n \in \omega}$ is st- $\tau_{\text {Arz }}$ convergent to $f$ on compacta, 
(iii) $\left(f_{n}\right)_{n \in \omega}$ is st- $\tau_{\square}$ convergent to $f$,

(iv) $\left(f_{n}\right)_{n \in \omega}$ is st- $\tau_{\mathrm{Al}}$ convergent to $f$.

We continue our analysis with respect to the statistical version of these convergences, and we prove that the whole statistical pointwise convergence in $C(X)$ ensures convergence in the two symmetric topologies of the decomposition of the sticking topology.

In [6] (Proposition 3.2), the author proved that if $\left(f_{n}\right)_{n \in \omega}$ is a sequence of functions in $C(X)$, that is $\tau_{p}$-convergent to $g \in \mathfrak{U}(X)$, then the sequence is $\tau^{u}$-convergent to $g$. And he shows that the $\tau_{p}$-convergence in $\mathcal{U}(\mathrm{X})$ does not ensure $\tau^{u}$-convergence, even if the limit is continuous and convergences are statistical. In the next proposition we clarify what exactly must be added to the $\tau_{p}^{u}$-convergence of a sequence of lower semicontinuous functions with an upper semicontinuous limit to ensure the $\tau^{u}$-convergence even from the statistical point of view.

Proposition 4.5. Let $\left(f_{n}\right)_{n \in \omega}$ be a sequence of functions in $\mathcal{L}(X)$. If $\left(f_{n}\right)_{n \in \omega}$ is st- $\tau_{p}^{u}$-convergent to $g \in \mathcal{U}(X)$ and $\left(f_{n}\right)_{n \in \omega}$ is st-almost below $g$ in $X$, then $\left(f_{n}\right)_{n \in \omega}$ is st- $\tau^{u}$ convergent to $g$.

Proof. Fix $F=\left\{x_{1}, \ldots, x_{n}\right\}$ a finite subset of $X$, and $\epsilon>0$. We show that there is $A \subset \mathbb{N}$ statistically dense such that for every $n \in A$ it follows that $f_{n} \in[F ; \epsilon]^{u}(g)$. Since $\left(f_{n}\right)_{n \in \omega}$ is st- $\tau_{p}^{u}$-convergent to $g$, there is $M \subset \mathbb{N}$ statistically dense, such that for every $x \in F$ and for all $n \in M$ we have $g(x) \leq f_{n}(x)+\epsilon / 3$. Let $n_{1} \in M$, since $f_{n_{1}}$ is lower semicontinuous at each $x_{i} \in F$; there exists $U_{x_{i}}$ such that for every $y \in U_{x_{i}}, f_{n_{1}}(y)>f_{n_{1}}\left(x_{i}\right)-\epsilon / 3$. By upper semicontinuity of $g$ at each $x_{i} \in F$, there exists $W_{x_{i}}$ such that for every $y \in W_{x_{i}}, g(y)<$ $g\left(x_{i}\right)+\epsilon / 3$. Also $\left(f_{n}\right)_{n \in \omega}$ is st-almost below $g$ at each $x_{i} \in F$; therefore for every $i \leq n$, there is $\delta_{i}>0$ such that for every $y \in S\left(x_{i}, \delta_{i}\right)$, there is $B_{y} \subset \mathbb{N}$ statistically dense, and for all $n \in B_{y}, f_{n}(y)<g\left(x_{i}\right)+\epsilon / 3$. Set $V=\bigcup_{i=1}^{n}\left(W_{x_{i}} \cap V_{x_{i}} \cap S\left(x_{i}, \delta_{i}\right)\right)$. For every $v \in V$ we have $g(v)<g\left(x_{i}\right)+\epsilon / 3<f_{n_{1}}\left(x_{i}\right)+2 \epsilon / 3<f_{n_{1}}(v)+\epsilon$. Let $A=\left(\bigcap_{i=1}^{n} B_{x_{i}}\right) \cap M$. For all $n \in A$ and each $x_{i} \in F$ it follows that $f_{n}\left(x_{i}\right) \leq g\left(x_{i}\right)+\epsilon / 3$ and $g\left(x_{i}\right)<f_{n}\left(x_{i}\right)+\epsilon$; thus $\left(f_{n}\right)_{n \in \omega}$ is statistically $\tau^{u}$-convergent to $g$.

We underline that there is an asymmetric role of the upper and lower decomposition of the pointwise convergence with respect to the upper and lower decomposition of the sticking topology. In fact with the same assumptions on the functions and the limit, the necessary condition to get the st- $\tau^{u}$ convergence is guaranteed by the st- $\tau^{l}$ convergence.

Proposition 4.6. Let $\left(f_{n}\right)_{n \in \omega}$ be a sequence of functions in $\mathcal{L}(X)$ and $g \in \mathcal{U}(X)$. If $\left(f_{n}\right)_{n \in \omega}$ is st- $\tau^{l}$ convergent to $g$, then

(i) $g$ is continuous,

(ii) $\left(f_{n}\right)_{n \in \omega}$ is st- $\tau_{p}^{u}$ convergent to $g$,

(iii) $\left(f_{n}\right)_{n \in \omega}$ is st-almost below $g$.

Proof. To prove $(i)$, it is sufficient to prove lower semicontinuity of $g$. Fix that $x_{0} \in X$ and $\epsilon>0$. By assumption there is $A \subset \mathbb{N}$ statistically dense, and for all $n \geq A, f_{n} \in\left[\left\{x_{0}\right\}, \epsilon / 3\right]^{l}(g)$. Thus there is $\delta>0$ such that for all $y \in S\left(x_{0}, \delta\right), g(y)>f_{n}(y)-\epsilon / 3$ and $\left|g\left(x_{0}\right)-f_{n}\left(x_{0}\right)\right|<\epsilon / 3$. 
Let $n_{0} \in A$. Since $f_{n_{0}}$ is lower semicontinuous, we may assume that for every $y \in S\left(x_{0}, \delta\right)$ we have also $f_{n_{0}}(y)>f_{n_{0}}\left(x_{0}\right)-\epsilon / 3$. It follows that for $y \in S\left(x_{0}, \delta\right)$,

$$
g\left(x_{0}\right)<f_{n_{0}}\left(x_{0}\right)+\frac{\epsilon}{3}<f_{n_{0}}(y)+\frac{2 \epsilon}{3}<g(y)+\epsilon .
$$

To prove (iii), fix $x_{0} \in X$ and $\epsilon>0$. Let $F=\left\{x_{0}\right\}$, since $\left(f_{n}\right)_{n \in \omega}$ statistically $\tau^{l}$ - converges to $g$; there is $A \subset \mathbb{N}$ statistically dense, $\delta_{1}>0$ such that for all $y \in S\left(x_{0}, \delta_{1}\right)$ and $n \in A$, we have $g(y)>f_{n}(y)-\epsilon / 2$ and $\left|g\left(x_{0}\right)-f_{n}\left(x_{0}\right)\right|<\epsilon / 2$. By upper semicontinuity of $g$ at each $x_{0}$ there exists $\delta_{0}>0$ such that for all $y \in S\left(x_{0}, \delta_{0}\right), g(y)<g\left(x_{0}\right)+\epsilon / 2$. Let $\delta=\min \left\{\delta_{0}, \delta_{1}\right\}$. For all $n \in A$ and $y \in S\left(x_{0}, \delta\right)$ it follows that $f_{n}(y) \leq g(y)+\epsilon / 2<g\left(x_{0}\right)+\epsilon$.

From Propositions 4.5 and 4.6 we have the following corollaries.

Corollary 4.7. Let $\left(f_{n}\right)_{n \in \omega}$ be a sequence of functions in $C(X)$ and $g \in \mathcal{L}(X)$. If $\left(f_{n}\right)_{n \in \omega}$ is st- $\tau^{u}$ convergent to $g$, then $\left(f_{n}\right)_{n \in \omega}$ is st- $\tau^{l}$ convergent to $g$.

Corollary 4.8. Let $\left(f_{n}\right)_{n \in \omega}$ be a sequence of functions in $C(X)$ and $g \in \mathcal{U}(X)$. If $\left(f_{n}\right)_{n \in \omega}$ is st- $\tau^{l}$ convergent to $g$, then $\left(f_{n}\right)_{n \in \omega}$ is st- $\tau^{u}$ convergent to $g$.

If we restrict our attention to continuous functions, we can relate results of the previous section due to the pointwise convergence in $\mathbb{R}^{X}$, namely, Corollary 3.7, to the sticking topology in $C(X)$.

Theorem 4.9. Let $\left(f_{n}\right)_{n \in \omega}$ be in $C(X)$. If $\left(f_{n}\right)_{n \in \omega}$ is st- $\tau_{p}$ convergent to $g$, then the following are equivalent:

(i) $g$ is continuous,

(ii) $\left(f_{n}\right)_{n \in \omega}$ is st-almost below and st-almost above $g$,

(iii) $\left(f_{n}\right)_{n \in \omega}$ is st-weakly exhaustive,

(iv) $\left(f_{n}\right)_{n \in \omega}$ is st- $\tau_{\square}$ convergent to $g$.

Combining Corollaries 4.7 and 4.8, we show that assuming the statistical pointwise convergence in $C(X)$, the convergence in one of the two symmetric topologies $\tau^{u}$ and $\tau^{l}$ automatically ensures convergence in the whole sticking topology, since they coincide.

Corollary 4.10. Let $\left(f_{n}\right)_{n \in \omega}$ and $g \in C(X)$. If $\left(f_{n}\right)_{n \in \omega}$ is st- $\tau_{p}$ convergent to $g$, then the following are equivalent:

(i) $\left(f_{n}\right)_{n \in \omega}$ is st- $\tau^{u}$ convergent to $g$,

(ii) $\left(f_{n}\right)_{n \in \omega}$ is st- $\tau^{l}$ convergent to $g$.

\section{Acknowledgments}

The paper is supported by GNSAGA. The author is very grateful to Professor Giuseppe Di Maio for the valuable comments and suggestions that have become improvements. The author also thanks the referees for the careful reading and suggestions. 


\section{References}

[1] A. Caserta and Lj. D. R. Kočinac, "On statistical exhaustiveness," . In press.

[2] A. Caserta, G. Di Maio, and Lj. D. R. Kočinac, "Statistical convergence in function spaces," . In press.

[3] M. K. Khan and C. Orhan, "Matrix characterization of A-statistical convergence," Journal of Mathematical Analysis and Applications, vol. 335, no. 1, pp. 406-417, 2007.

[4] N. Bouleau, "Une structure uniforme sur un espace F(E, F)," Cahiers de Topologie et Géométrie Différentielle Catégoriques, vol. 11, pp. 207-214, 1969.

[5] N. Bouleau, "On the coarsest topology preserving continuity," General Topology. In press, http:/ /arxiv .org/abs/math/0610373v1.

[6] G. Beer, "Semicontinuous limits of nets of continuous functions," . In press.

[7] W. J. Pervin, Foundations of General Topology, Academic Press, New York, NY, USA, 1964.

[8] W. J. Pervin, “Quasi-uniformization of topological spaces," Mathematische Annalen, vol. 147, pp. 316317, 1962.

[9] P. Fletcher and W. F. Lindgren, Quasi-Uniform Spaces, vol. 77 of Lecture Notes in Pure and Applied Mathematics, Marcel Dekker, New York, NY, USA, 1982.

[10] J. L. Kelley, General Topology, D. Van Nostrand Company, Princeton, NJ, USA, 1955.

[11] A. Zygmund, Trigonometric Series, Cambridge University Press, Cambridge, UK, 2nd edition, 1979.

[12] H. Fast, "Sur la convergence statistique," Colloquium Mathematicum, vol. 2, pp. 241-244, 1951.

[13] R. C. Buck, "Generalized asymptotic density," American Journal of Mathematics, vol. 75, pp. 335-346, 1953.

[14] G. Di Maio and L. D. R. Kočinac, "Statistical convergence in topology," Topology and its Applications, vol. 156, no. 1, pp. 28-45, 2008.

[15] J. A. Fridy, “On statistical convergence," Analysis, vol. 5, no. 4, pp. 301-313, 1985.

[16] T. Šalát, "On statistically convergent sequences of real numbers," Mathematica Slovaca, vol. 30, no. 2, pp. 139-150, 1980.

[17] H. Çakalli and M. K. Khan, "Summability in topological spaces," Applied Mathematics Letters, vol. 24, no. 3, pp. 348-352, 2011.

[18] V. Gregoriades and N. Papanastassiou, "The notion of exhaustiveness and Ascoli-type theorems," Topology and its Applications, vol. 155, no. 10, pp. 1111-1128, 2008.

[19] G. Beer and S. Levi, "Strong uniform continuity," Journal of Mathematical Analysis and Applications, vol. 350, no. 2, pp. 568-589, 2009.

[20] A. Caserta, G. Di Maio, and L. Holá, "Arzelà's theorem and strong uniform convergence on bornologies," Journal of Mathematical Analysis and Applications, vol. 371, no. 1, pp. 384-392, 2010.

[21] P. S. Alexandroff, Einführung in die Mengenlehre und die Theorie der Reellen Funktionen, Deutscher Verlag der Wissenschaften, Berlin, Germany, 1956.

[22] C. Arzelà, "Intorno alla continuà della somma di infinite funzioni continue," Rend. R. Accad. d. Scienze dell'Istituto di Bologna, pp. 79-84, 1884.

[23] R. G. Bartle, "On compactness in functional analysis," Transactions of the American Mathematical Society, vol. 79, pp. 35-57, 1955. 


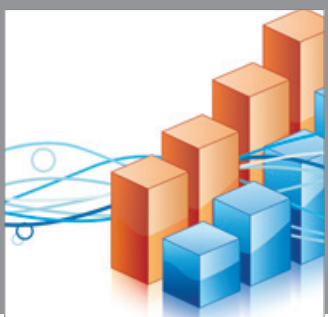

Advances in

Operations Research

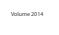

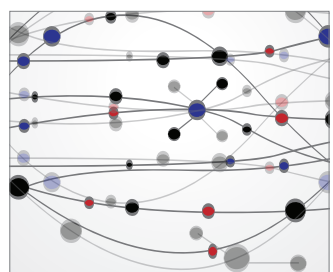

\section{The Scientific} World Journal
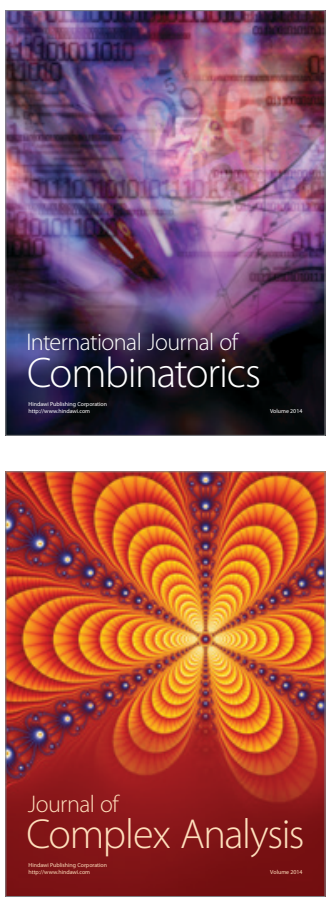

International Journal of

Mathematics and

Mathematical

Sciences
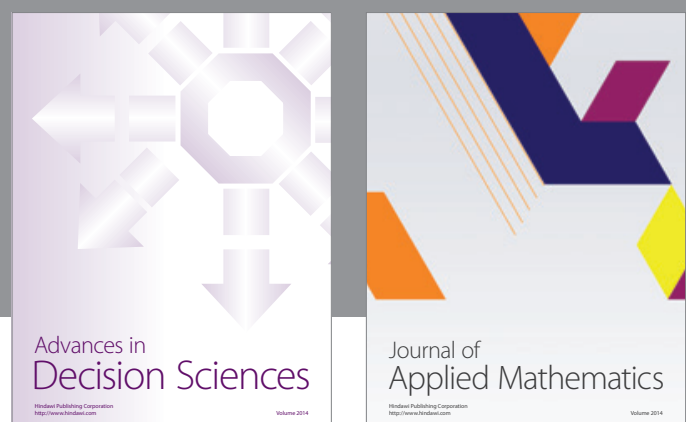

Journal of

Applied Mathematics
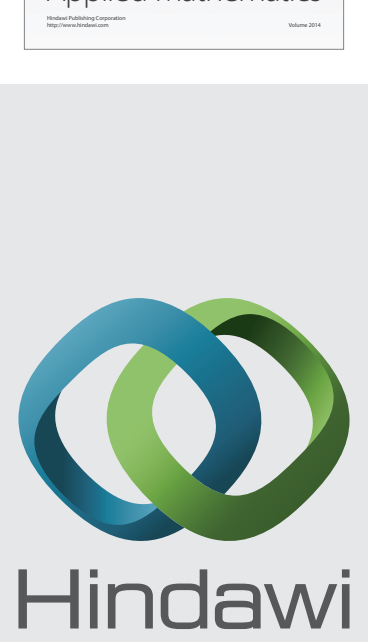

Submit your manuscripts at http://www.hindawi.com
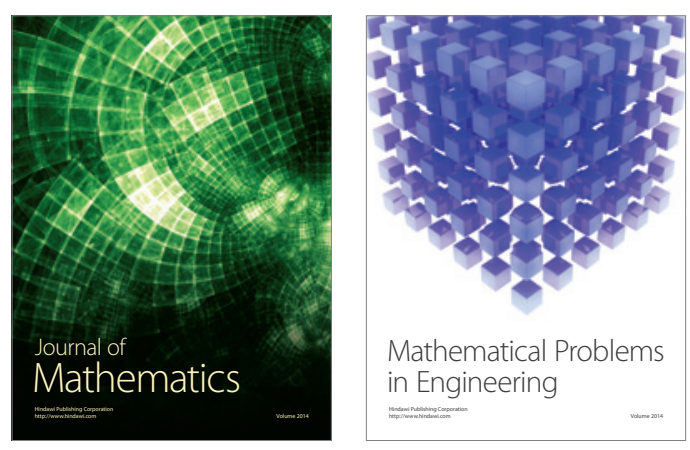

Mathematical Problems in Engineering
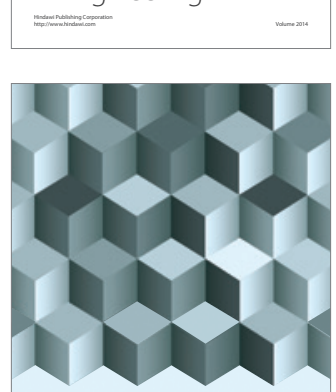

Journal of

Function Spaces
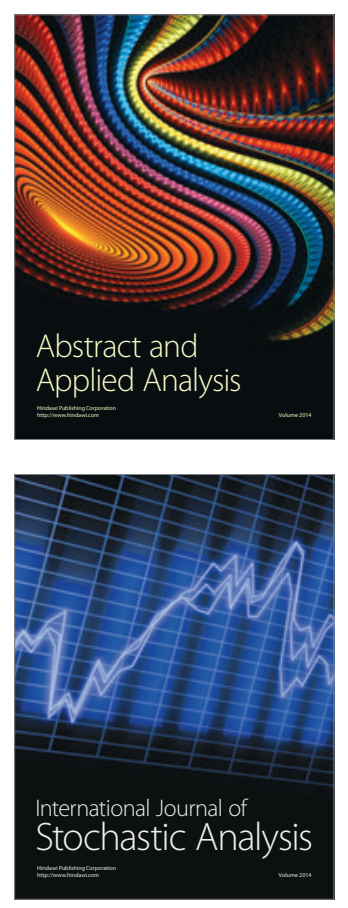

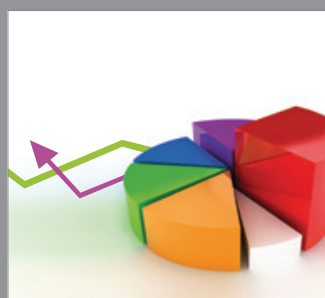

ournal of

Probability and Statistics

Promensencen
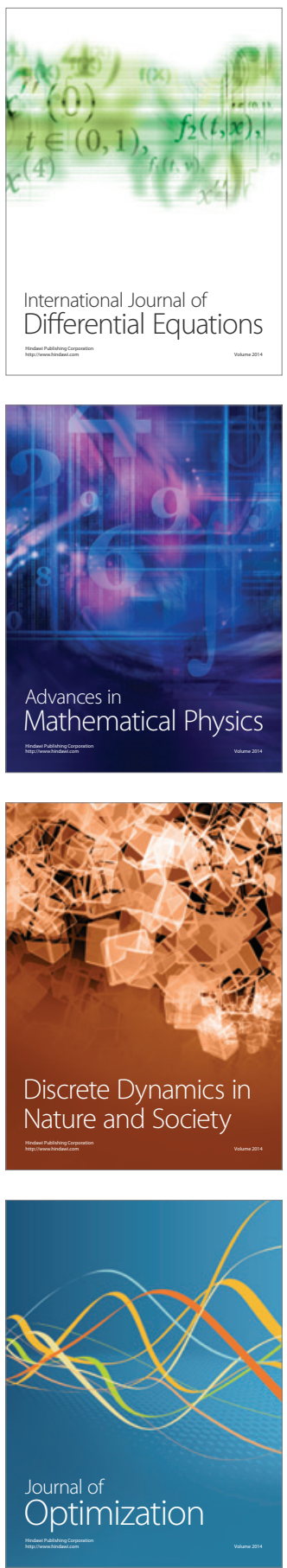